\title{
Test-bolus versus bolus-tracking in the timing of hepatic arterial phase at contrast-enhanced magnetic resonance imaging in patients with hepatocellular carcinoma
}

\author{
Emilio Quaia* \\ Department of Medical, Surgical and Health Sciences, Operative Unit of Radiology, Cattinara Hospital, Italy
}

\begin{abstract}
Aim: To evaluate test-bolus versus bolus-tracking in the timing of hepatic arterial phase at contrast-enhanced magnetic resonance (MR) imaging in patients with hepatocellular carcinoma (HCC).

Methods: Eighteen patients with HCC were scanned by contrast-enhanced MR imaging with a delay time for the hepatic arterial phase calculated by test - bolus ( $\mathrm{n}=9$ patients) or bolus - tracking ( $\mathrm{n}=9$ patients)

Results: Test-bolus and bolus-tracking techniques did not differ in SNR (301.2 \pm 154.54 vs. $330.77 \pm 240.75 ; \mathrm{P}=0.89)$ and CNR (24.5 \pm 18.74 vs. $19.24 \pm 13.67$; $\mathrm{P}=0.89$ ).

Conclusions: Test - bolus vs bolus - tracking did not differ in the timing of hepatic arterial phase in patients with HCC.
\end{abstract}

\section{Introduction}

Hypervascularity on hepatic arterial phase after contast injection is one of the essential feature for the diagnosis of HCC according to the American Association for the Study of Liver Disease (ASL) [1] and the European Association for the study of the liver (EASL) [2] even though it is a transient phenomenon, and errors of only a few seconds during the image acquisition on hepatic arterial phase may determine a reduced diagnostic confidence and accuracy. Anyway, the determination of the precise timing of optimal contrast enhancement for detection of hepatocellular carcinoma (HCC) is technically challenging because this timing is affected by the patient's circulation, the contrast injection protocol, and the imaging protocol.

Two methods - arbitrary fixed delay and test - bolus - have been used traditionally to determine the delay time from the start of contrast injection for the hepatic arterial phase at MR imaging. With the arbitrary fixed delay method, MR imaging is initiated 15-20 seconds after the start of the injection. This technique does not take into account injection- or patient-related variables. More properly, with the test-bolus method, $1-2 \mathrm{~mL}$ of contrast medium are injected and simultaneous rapid and repetitive MR imaging at one level is performed during free breathing to determine the time to peak aortic enhancement. The delay time is calculated on the basis of the time of peak aortic enhancement, injection volume, and rate. While considered the most accurate, this method requires additional imaging and calculations.

A further method corresponds to real-time bolus-tracking providing hepatic arterial phase images tailored to an individual patient's cardiac output and peripheral bloodstream kinetic. A real time bolus-triggered method includes breath-hold instructions initiated as the contrast bolus reaches the celiac trunk (trigger point), and imaging initiated at an 5-10 s delay from the trigger point. This method is very used despite it presents some dependence on the operator's subjective assessment of the contrast arrival in the abdominal aorta.

To our knowledge, only few previous studies [3-5] have been conducted to evaluate the optimal acquisition delay for dynamic contrast-enhanced multiphase MR imaging of the liver and HCC even though no further study compared test - bolus and bolus - tracking techniques.

The aim of this study was to evaluate test-bolus versus bolustracking in the timing of hepatic arterial phase at contrast-enhanced MR imaging in patients with HCC.

\section{Materials and methods}

\section{Patients}

The institutional review board of our hospital approved this singlecentre prospective observational study. All patients provided informed content.

We included all patients who met the following inclusion criteria: biopsy-proven diagnosis of liver cirrhosis due to type $\mathrm{B}$, type $\mathrm{C}$, or alcoholic hepatitis; from one to four hypervascular nodules (diameter,

Correspondence to: Emilio Quaia, Associate Professor of Radiology, Department of Medical, Surgical and Health Sciences, Operative Unit of Radiology, Cattinara Hospital, Strada di Fiume 447, 34149, Trieste, Italy, E-mail: quaia@units.it

Key words: liver, hepatocellular, carcinoma, test-bolus, bolustracking

Received: October 04, 2016; Accepted: November 17, 2016; Published: November 21, 2016 
Quaia E (2016) Test-bolus versus bolus-tracking in the timing of hepatic arterial phase at contrast-enhanced magnetic resonance imaging in patients with hepatocellular carcinoma

$2-4 \mathrm{~cm}$; mean $\pm \mathrm{SD}, 3.5 \pm 1.2)$ suspected for HCC at contrast-enhanced $\mathrm{CT}$ and referred to contrast-enhanced abdominal MR imaging to improve the diagnostic confidence in the characterization of the dominant HCC nodule(s) or to identify additional nodules; no history of hepatic surgery or thermal ablation or chemoembolization; absence of renal failure (serum creatinine concentration $<1.5 \mathrm{mg} / \mathrm{dL}$ ); contraindication to use of gadolinium-based contrast material. We initially included 25 patients while seven patients were excluded: three had tumor thrombi in the central portal vein, and four had numerous tumors involving the entire liver that may have changed hepatic hemodynamics.

Thus the final study sample consisted in 18 patients $(8$ male and 10 female; mean age, 66 years \pm 12 ; body weight range, $33-69 \mathrm{~kg}$; mean, $55.0 \pm 8.4$ [SD] kg). The age range of men was $48-88$ years (mean, 67.5 years), and that of the women was 52-85 years (mean, 71.0 years). The definitive diagnosis of HCC was based on histology $(n=2)$, histopathologic evidence after hepatic surgery $(n=3)$, on imaging follow-up based on contrast-enhanced dynamic CT showing an increase in tumor size within 3 months $(n=2)$, or on typical enhancement pattern on contrast-enhanced MR imaging $(n=11$ patients) based on EASL [1] or ASL criteria [2].

\section{MR imaging}

The MR imaging examination was performed within one month from contrast-enhanced CT using a superconducting magnet operating at $1.5 \mathrm{~T}$ (Achieva, 1,5T release 2.1.3.4, Philips Healthcare, Best, The Netherlands) with a peak gradient amplitude of $30 \mathrm{mT} / \mathrm{m}$ and a peak slew rate of $150 \mathrm{~T} / \mathrm{m} / \mathrm{sec}$. Images were acquired in the transverse plane with a combined four-channel anteroposterior phased-array surface coil. Parallel imaging with a sensitivity-encoding (SENSE) technique with a factor of 1.5 - 1.7 was employed. A three-quarter field of view was used in the phase-encoding direction. Presaturation pulses were applied above and below the imaging volume to diminish flow artifacts.

The baseline MR imaging examination included a breath-hold fast spin-echo T2-weighted MR imaging sequence, a fat-suppressed T2weighted sequence, a T1-weighted in-phase and out-of-phase sequence, and a fast-field echo T2-weighted sequence. Dynamic MR imaging was performed before and after gadobenate dimeglumine (Gd-BOPTA, 0.1 $\mathrm{mmol} / \mathrm{kg} ; 2 \mathrm{~mL} / \mathrm{sec}$ ) injection via a forearm or antecubital vein at $2 \mathrm{~mL} /$ sec through a 18-gauge intravenous catheter employing an automated injector (Spectris MR Injector; Medrad, Indianola, Pa), followed by $20 \mathrm{~mL}$ of saline at $2 \mathrm{~mL} / \mathrm{sec}$. Each dynamic contrast-enhanced MR imaging examination consisted in T1-weighted breath-hold resolution isotropic high volume (THRIVE) 3D with fat suppression MR sequences, with centric k-space acquisition, acquired before and after contrast administration at hepatic arterial, portal venous (70 seconds), equilibrium ( 3 minutes) and hepato-biliary phase ( 2 hours from the beginning of contrast injection).

The delay time after i.v. contrast injection for the hepatic arterial phase was calculated by test - bolus in 9 patients by injecting $2 \mathrm{~mL}$ of contrast followed by $20 \mathrm{~mL}$ saline flush to determine the time to peak enhancement in the liver parenchyma according to the formula: $1 / 2$ time of injection + time to the peak $-1 / 2$ acquisition time +4 seconds.

In the remaining 9 patients the delay time for the arterial phase was calculated by bolus - tracking technique. Between the precontrast and dynamic image acquisitions, an MR fluoroscopic sequence for contrast bolus chase (TR/TE, $4 / 0.87 \mathrm{msecs}$, flip angle $40^{\circ}$, slice thickness $80 \mathrm{~mm}$, field-of-view $530 \times 530 \mathrm{~mm}$ with a matrix of $256 \times 128$, acquisition time
0.512 seconds) was performed, and yielded a subtracted coronal twodimensional projection of the abdominal aorta every second. Dynamic sequence on hepatic arterial phase began with 5 seconds delay after visualization of contrast at the abdominal aorta. Portal venous and equilibrium phase MR images were acquired with 70 seconds and 3 minutes delay from the beginning of contrast injection while hepatobiliary phase (HBP) MR images were acquired 2 hours from contrast agent injection.

\section{Qualitative evaluation}

To compare image quality between the hepatic arterial phase images acquired by the two techniques, two board-certified diagnostic radiologists with 22 and 11 years of experience in abdominal MR imaging performed independent visual assessments of image noise. They were blinded to clinical data and evaluated arterial and portal venous phase images without access to images obtained in other phases. After their independent evaluations, the reviewers assigned a score in consensus. Image noise was quantified on a 3-point scale on which a score of 3 denoted almost no or minimal image noise throughout the image; 2, a moderate degree of image noise that did not affect diagnostic utility, and 1 , severe image noise that may severely hamper diagnostic utility. All readings were performed on a PACS- integrated workstation (19-inch TFT display, resolution $2560 \times 1600$ pixels, Ebit Sanità AET, Genoa, Italy) by using a proprietary software package (Ebit Sanità AET, Genoa, Italy).

\section{Quantitative analysis}

The same two readers, consensually, calculated the signal-tonoise ratio (SNR $=$ mean SI liver $/ \mathrm{SD}$ ) and contrast-to-noise ratio $(\mathrm{CNR}=$ mean SI lesion - mean SI liver/SD) by outlining freehand polygonal region of interest (ROIs) on the site of the hypervascular nodule and on the adjacent liver parenchyma within $2 \mathrm{~cm}$ from the lesion on images acquired during the hepatic arterial phase by using a dedicated workstation (Extended WorkSpace eXplore, Philips, Best, The Netherlands).

\section{Statistical analysis}

Statistical analysis was performed using STATA, version 13 (StataCorp, Lakeway Drive, College Station, Texas, USA). We used the non-parametric correlation analysis and the intra-class correlation coefficient (ICC) to assess the agreement between test - bolus and bolus - tracking technique. Inter-observer agreement was considered as slight for an ICC of $<0.21$, fair for a value of $0.21-0.40$, moderate for a value of $0.41-0.60$, good for a value of $0.61-0.80$, and optimal for a value of $0.81-1.00$.

For all tests a $\mathrm{P}$ value $<.05$ was considered to indicate a statistically significant difference.

\section{Results}

Tables 1 show the results of SNR and CNR of test - bolus and bolus - tracking techniques.

Test - bolus and bolus - tracking techniques did not differ in the estimation of the correct delay time for the hepatic arterial phase at contrast-enhanced MR imaging in patients with HCC in liver cirrhosis. There was good correlation $(\mathrm{r}=0.85)$ and agreement $(\mathrm{ICC}=0.9)$ between the delay times (25-27 seconds) for the hepatic arterial phase calculated by test - bolus and bolus - tracking techniques. Test - bolus versus bolus - tracking techniques did not differ both in SNR (301.2 \pm 154.54 vs. $330.77 \pm 240.75 ; \mathrm{P}=0.89$ Mann-Whitney $\mathrm{U}$ test $)$ and CNR (24.5 \pm 
Quaia E (2016) Test-bolus versus bolus-tracking in the timing of hepatic arterial phase at contrast-enhanced magnetic resonance imaging in patients with hepatocellular carcinoma

Table 1a. Test - Bolus data

\begin{tabular}{|c|c|c|c|c|c|}
\hline Patient & ROI Liver & ROI Lesion & ROI Air & SNR & CNR \\
\hline 1 & $640 \pm 96.6$ & $802.4 \pm 10$ & $14.5 \pm 17.2$ & 162.4 & 11.2 \\
\hline 2 & $869.4 \pm 10.4$ & $1041.1 \pm 88.9$ & $20.1 \pm 12.8$ & 171.7 & 8.54 \\
\hline 3 & $597.5 \pm 72.2$ & $989.8 \pm 30.9$ & $1.5 \pm 9.8$ & 392.3 & 41.29 \\
\hline 4 & $762 \pm 32.3$ & $1278.2 \pm 60.9$ & $14.4 \pm 16.6$ & 516.2 & 35.84 \\
\hline 5 & $450.6 \pm 26.6$ & $760 \pm 54.9$ & $12.3 \pm 16.8$ & 309.4 & 25.15 \\
\hline 6 & $715.9 \pm 31.1$ & $1020 \pm 59.6$ & $21 \pm 16.8$ & 304.1 & 14.48 \\
\hline 7 & $564.1 \pm 54.3$ & $664.7 \pm 26.3$ & $14 \pm 9.3$ & 100.6 & 7.18 \\
\hline 8 & $769.2 \pm 65.6$ & $990.2 \pm 21.2$ & $15.7 \pm 11.8$ & 221 & 14.07 \\
\hline 9 & $201.9 \pm 5.6$ & $735 \pm 11$ & $8.5 \pm 12.3$ & 533.1 & 62.71 \\
\hline Mean \pm SD & $618.96 \pm 200.42$ & $920.16 \pm 194.16$ & $13.71 \pm 3.17$ & $301.2 \pm 154.54$ & $24.5 \pm 18.74$ \\
\hline
\end{tabular}

Table 1b. Bolus - Tracking data.

\begin{tabular}{|c|c|c|c|c|c|}
\hline Patient & ROI Liver & ROI Lesion & ROI Air & SNR & CNR \\
\hline 10 & $428.4 \pm 80.6$ & $604.2 \pm 70.2$ & $11.9 \pm 10.3$ & 175.8 & 14.77 \\
\hline 11 & $484 \pm 96.2$ & $811.40 \pm 17.6$ & $19.6 \pm 11.8$ & 327.4 & 16.7 \\
\hline 12 & $1032.7 \pm 53.7$ & $1270.6 \pm 86.6$ & $16.1 \pm 22.3$ & 237,9 & 14.77 \\
\hline 13 & $1003.8 \pm 37.9$ & $1041 \pm 92.3$ & $15.1 \pm 13.1$ & 37.2 & 2.46 \\
\hline 14 & $772.6 \pm 28.1$ & $1427 \pm 28.8$ & $20.5 \pm 7.1$ & 654.4 & 31.92 \\
\hline 15 & $705.7 \pm 61.2$ & $1468.8 \pm 66.3$ & $16.3 \pm 9.5$ & 763.1 & 46.81 \\
\hline 16 & $527.5 \pm 21.6$ & $636.8 \pm 34.7$ & $23.9 \pm 18$ & 109.3 & 4.57 \\
\hline 17 & $866 \pm 45.6$ & $1156.5 \pm 35.8$ & $17.2 \pm 13$ & 290.5 & 16.88 \\
\hline 18 & $276.8 \pm 14.7$ & $658.1 \pm 13.2$ & $15.7 \pm 7.5$ & 381.3 & 24.28 \\
\hline Mean \pm SD & $677.5 \pm 264.7$ & $1008.27 \pm 343.13$ & $17.37 \pm 3.5$ & $330.77 \pm 240.75$ & $19.24 \pm 13.67$ \\
\hline
\end{tabular}

18.74 vs. $19.24 \pm 13.67 ; \mathrm{P}=0.89)$.

\section{Discussion}

The first finding of our study was that test - bolus and bolus - tracking techniques did not differ in the estimation of the correct delay time for the hepatic arterial phase at contrast-enhanced MR imaging to visualize hypervascular HCC nodules in a background of liver cirrhosis. The test - bolus and bolus - tracking techniques present a good correlation and agreement for the timing of hepatic arterial phase. Bolus chase may be applied in the place of the bolus test, for those sequences with an early filling of the center of the K-space, to calculate the correct delay time for the hepatic arterial phase at contrast-enhanced MR imaging. This study shows evidence that accurate capture of liver arterial phase can be attributed mostly to the ability of real-time bolus tracking to resolve the strong variance in contrast arrival time into the aorta.

Theoretically, test - bolus technique provides the most accurate determination of the acquisition delay time for the hepatic arterial phase. The use of fluoroscopic triggering is appropriate only for MR sequences with which the high-contrast central portion of $\mathrm{k}$-space is filled first, at the beginning of the acquisition, as in the present study. Contrary to the 3D THRIVE sequences with centric K-space acqusition used in this study, conventional 3D gradient-recalled echo sequences typically employ a sequential $\mathrm{k}$-space acquisition with data acquired linearly or radially over many segments while the center of $\mathrm{k}$-space is passed multiple times over the scan duration. As a consequence this averages the dynamic image contrast, such that the middle of the acquisition is representative of the peak contrast on the MR images. For these methods of K-space acquisition the test - bolus technique remains the most appropriate technique to be used in the calculation of the timing for the correct hepatic arterial phase in cirrhotic patients with HCC.
The second finding of the present study was the absence of difference between the SNR and CNR of HCC between test - bolus and bolus - tracking techniques. This can allow to use undifferently the two timing techniques if centric K-space acquisition is employed in dynamic contrast-enhanced MR imaging.

The main limitation of the present study is that an independent patient sample analysis was performed instead of paired data analysis with intra-individual comparison.

In conclusion test - bolus $v s$. bolus - tracking did not differ in the timing of hepatic arterial phase in patients with HCC.

\section{References}

1. Bruix J, Sherman M; American Association for the Study of Liver Diseases (2011) Management of hepatocellular carcinoma: an update. Hepatology 53: 1020-1022. [Crossref]

2. European Association for the Study of the Liver, European Organisation for Research and Treatment of Cancer ( 2012) EASL-EORTC Clinical Practice Guidelines: Management of hepatocellular carcinoma. J Hepatol 56: 908-943. [Crossref]

3. Goshima S, Kanematsu M, Kondo H, Shiratori Y, Onozuka M, et at. ( 2009) Optimal acquisition delay for dynamic contrast-enhanced MRI of hypervascular hepatocellular carcinoma. AJR Am J Roentgenol 192: 686-692. [Crossref]

4. Haradome H, Grazioli L (2012) Gadoxetic Acid Disodium-Enhanced Hepatocyte Phase MRI: Can Increase the Flip Angle Improve Focal Liver Lesion Detection? J Magn Reson Imaging 35: 132-139. [Crossref]

5. Mori K, Yoshioka H, Takahashi N (2005) Triple arterial phase dynamic MRI with sensitivity encoding for hypervascular hepatocellular carcinoma: comparison of the diagnostic accuracy among the early, middle, late, and whole triple arterial phase imaging. AJR 184: 63-69.

6. Yoshioka H, Takahashi N, Yamaguchi M, Lou D, Saida Y, et al. (2002) Double arterial phase dynamic MRI with sensitivity encoding (SENSE) for hypervascular hepatocellular carcinomas. J Magn Reson Imaging 16: 259-266. [Crossref]

7. Kanematsu M, Semelka RC, Matsuo M, (2002) Gadolinium-enhanced MR imaging of the liver: optimizing imaging delay for hepatic arterial and portal venous phases - a 
Quaia E (2016) Test-bolus versus bolus-tracking in the timing of hepatic arterial phase at contrast-enhanced magnetic resonance imaging in patients with hepatocellular carcinoma

prospective randomized study in patients with chronic liver damage. Radiology 225: 407-415. [Crossref]

8. Hussain HK, Londy FJ, Francis IR, Nghiem HV, Weadock WJ, et al. (2003) Hepatic Arterial Phase MR Imaging with Automated Bolus-Detection Threedimensional Fast
Gradient- Recalled-Echo Sequence: Comparison with Test-Bolus Method. Radiology 226: 558-566. [Crossref]

9. Sharma P, Kalb B, Kitajima HD, Salman KN, Burrow B, et al. (2011) Optimization of Single Injection Liver Arterial Phase Gadolinium Enhanced MRI Using Bolus Track Real-Time Imaging. J Magn Reson Imaging 33: 110-118.[Crossref]

Copyright: (C2016 Quaia E. This is an open-access article distributed under the terms of the Creative Commons Attribution License, which permits unrestricted use, distribution, and reproduction in any medium, provided the original author and source are credited. 